\title{
Gibbs Ensembles of Nonintersecting Paths
}

\author{
Alexei Borodin and Senya Shlosman \\ Caltech, Pasadena, USA \\ borodin@caltech.edu; \\ Centre de Physique Theorique, CNRS, \\ Luminy, Marseille, France \\ shlosman@cpt.univ-mrs.fr \\ and IITP, RAS, Moscow, Russia \\ shlosman@iitp.ru
}

August 7, 2018

\begin{abstract}
We consider a family of determinantal random point processes on the two-dimensional lattice and prove that members of our family can be interpreted as a kind of Gibbs ensembles of nonintersecting paths. Examples include probability measures on lozenge and domino tilings of the plane, some of which are non-translation-invariant.

The correlation kernels of our processes can be viewed as extensions of the discrete sine kernel, and we show that the Gibbs property is a consequence of simple linear relations satisfied by these kernels. The processes depend on infinitely many parameters, which are closely related to parametrization of totally positive Toeplitz matrices.
\end{abstract}

\section{Introduction}

It is well known that the Gibbs random fields are defined via prescribing their conditional distributions. In the case of the nearest-neighbor interactions these distributions are given by some relatively simple relations. The 
computation of their correlation functions is on the other hand usually a very difficult problem, because it requires the passing to the thermodynamic limit. In comparison, the determinantal random fields (or random point processes, both terms are used) are defined in such a way that the correlation functions are given by relatively simple direct formulas, while the computation of the conditional distributions may again require taking the thermodynamic limit, since the dependence is usually long-range.

The purpose of the present paper is the study of some 2D random fields $n=\left\{n_{t}=0,1, t \in \mathbb{Z}^{2}\right\}$, which are both determinantal and have in addition some kind of the Gibbs property. We wanted to understand which properties of the kernel produce the Gibbsianity of the random field. It turns out that the property sought is some linear relation on the matrix elements of the kernel. Below we explain this statement for a certain class of $2 \mathrm{D}$ determinantal random fields.

Every random field is specified by the (consistent) assignment of the probabilities $\operatorname{Pr}$ to the events $\left\{n_{t_{i}}=1, n_{t_{j}}=0\right\}$, for any two non-intersecting finite sets $\left\{t_{i}\right\},\left\{t_{j}\right\}$. We say that a random field $n$ is a determinantal random field with the kernel $K\left(t^{\prime}, t^{\prime \prime}\right)$, if for every finite collection $t_{i} \in \mathbb{Z}^{2}$

$$
\operatorname{Pr}_{K}\left\{n_{t_{i}}=1\right\}=\operatorname{det}\left\|K\left(t_{i}, t_{j}\right)\right\|
$$

The inclusion-exclusion principle then implies that the probability of a more general event

$$
\operatorname{Pr}_{K}\left\{n_{t_{i}}=1, n_{t_{j}}=0\right\}=(-1)^{h} \operatorname{det}\left\|\tilde{K}\left(t_{i}, t_{j}\right)\right\|,
$$

where

$$
\tilde{K}\left(t_{i}, t_{j}\right)=\left\{\begin{array}{lc}
K\left(t_{i}, t_{j}\right) & \text { if } t_{i} \neq t_{j} \\
K\left(t_{i}, t_{i}\right) & \text { if } t_{i}=t_{j} \text { and } n_{t_{i}}=1 \\
K\left(t_{j}, t_{j}\right)-1 & \text { if } t_{i}=t_{j} \text { and } n_{t_{j}}=0
\end{array}\right.
$$

and $h$ is the number of "holes", i.e. indices $\left\{t_{j}: n_{t_{j}}=0\right\}$. We will refer to the sites with values 1 as "particles". The above formula is sometimes referred to as complementation principle, cf. A.3 of [3].

In this paper we study random fields $n$, corresponding to the kernels $K$ constructed as follows. Suppose that for every $k \in \mathbb{Z}^{1}$ the function $\psi_{k}(u)$ is given, which can be one of the following four functions:

$$
\left(1-\alpha_{k}^{+} u\right)^{-1}, \quad\left(1-\alpha_{k}^{-} u^{-1}\right)^{-1}, \quad\left(1+\beta_{k}^{+} u\right), \quad\left(1+\beta_{k}^{-} u^{-1}\right),
$$


with positive constants $\alpha^{ \pm}, \beta^{ \pm}$. Let us also fix a complex number $z$ with $\Im z>0$ and denote $C_{ \pm}$any contour that joins $\bar{z}$ and $z$ and crosses the real axis at a point of $\mathbb{R}_{ \pm}$.

For $t^{\prime}=(\sigma, x), t^{\prime \prime}=(\tau, y)$ we define

$$
K(\sigma, x ; \tau, y) \equiv K_{\sigma, \tau}(x-y)= \begin{cases}\frac{1}{2 \pi i} \int_{C_{+}}\left(\prod_{k=\sigma+1}^{\tau} \psi_{k}(u)\right)^{-1} \frac{d u}{u^{x-y+1}} & \text { if } \sigma<\tau, \\ \frac{1}{2 \pi i} \int_{C_{+}} \frac{d u}{u^{x-y+1}} & \text { if } \sigma=\tau, \\ \frac{1}{2 \pi i} \int_{C_{-}} \prod_{k=\tau+1}^{\sigma} \psi_{k}(u) \frac{d u}{u^{x-y+1}} & \text { if } \sigma>\tau .\end{cases}
$$

Our main results concerning such determinantal random fields are threefold:

1. Due to the (particles or holes) interlacing property of our fields $n$, they can be interpreted as ensembles of non-intersecting infinite random lattice paths. (These ensembles are different for different kernels, and will be described in detail below.)

2. The collections $\omega=\left\{\omega_{i}\right\}$ of random lattice paths thus obtained are "Gibbs random paths ensembles". They are defined by the action functional $S_{K}(\omega)$, which is local and is determined by the parameters of the kernel $K$.

3. The validity of the above two statements follows from simple linear relations that the matrix elements of the kernel $K$ satisfy, and do not depend on $K$ otherwise.

\subsection{Gibbs Path Ensembles}

We will define the Gibbs random path ensemble, corresponding to the additive functional $S$. Here $S$ is a function defined on the set of all finite selfavoiding lattice paths $\omega$, which has the additivity property: if $\omega=\omega_{1} \cup \omega_{2}$, $\omega_{1} \cap \omega_{2}=\{t\} \in \omega$, then $S(\omega)=S\left(\omega_{1}\right)+S\left(\omega_{2}\right)$. Let $\mu$ be a probability 
distribution on the set of families of non-intersecting double-infinite polygonal lattice paths $\omega=\left\{\omega_{i},-\infty<i<\infty\right\}$. Let $\Lambda \subset \mathbb{Z}^{2}$ be a finite box, i.e. a finite connected subset of $\mathbb{Z}^{2}$ with connected complement. Let the paths $\omega_{i}$ be fixed outside $\Lambda$. We denote the restriction of $\omega$ to the complement of $\Lambda$ by $\omega_{\bar{\Lambda}}$. Some of the paths from $\omega$ are entering and exiting $\Lambda$. Let $P$ be the set $p_{1}, \ldots, p_{k} \in \partial \Lambda$ of all the entrance points to $\Lambda$, while $Q$ be the set of the exit points $q_{1}, \ldots, q_{k} \in \partial \Lambda$ from $\Lambda$. Let $\varrho_{\Lambda}=\varrho_{1}, \ldots, \varrho_{k}$ be a collection of non-intersecting lattice paths contained in $\Lambda$, joining the points $p_{1}, \ldots, p_{k}$ and $q_{1}, \ldots, q_{k}$. We denote the set of such $k$-tuples of paths by $\Omega_{\Lambda}(P, Q)$.

We say that the measure $\mu$ is a Gibbs measure with the action functional $S$, if for every triple $(\Lambda, P, Q)$ the conditional distributions of $\mu$ satisfy

$$
\mu\left(\varrho_{\Lambda} \mid \omega_{\bar{\Lambda}}\right)=\frac{\exp \left\{S\left(\varrho_{1}\right)+\ldots+S\left(\varrho_{k}\right)\right\}}{Z(\Lambda, P, Q)},
$$

where $Z(\Lambda, P, Q)=\sum_{\varrho_{\Lambda} \in \Omega_{\Lambda}(P, Q)} \exp \left\{S\left(\varrho_{1}\right)+\ldots+S\left(\varrho_{k}\right)\right\}$ is the partition function.

\section{$1.2 \quad$ The Interlacing Property}

This property of the random field $n$ holds almost surely with respect to the measure $\mathbf{P r}_{K}$, as we will show below. Its exact formulation is different at different locations and depends on the structure of the kernel $K$ at this location. The picture on Fig. 1 illustrates the various cases which are described below.

If for some $k$ we have $\psi_{k}(u)=\left(1-\alpha_{k}^{+} u\right)^{-1}$, then in the strip $R_{k}=$ $\left\{(\sigma, x) \in \mathbb{Z}^{2}: \sigma=k, k+1\right\}$ the following property holds $\mathbf{P r}_{K^{-}}$a.s.: For any two particles $n_{\left(k, x_{1}\right)}=n_{\left(k, x_{2}\right)}=1, x_{1}<x_{2}$, of the configuration $n$, separated by string of holes, $n_{(k, x)}=0$ for all $x_{1}<x<x_{2}$, we find on the neighboring line $\sigma=k+1$ exactly one particle $n_{(k+1, x)}=1$ sitting in the set $\left\{(k+1, x): x_{1} \leq x<x_{2}\right\}$, and the rest of points of this set host holes. This is the upward interlacing of particles.

Consider now the correspondence $\pi^{+}$, which assigns to a particle $n_{(k, x)}=1$ the particle $n_{\left(k+1, \pi^{+}(x)\right)}=1$, where $\pi^{+}(x)=\min \left\{y \geq x: n_{(k+1, y)}=1\right\} \geq x$. The correspondence $\pi^{+}$is one-to-one with probability one. Let us connect each particle $n_{(k, x)}=1$ with the corresponding particle $n_{\left(k+1, \pi^{+}(x)\right)}=1$ by 


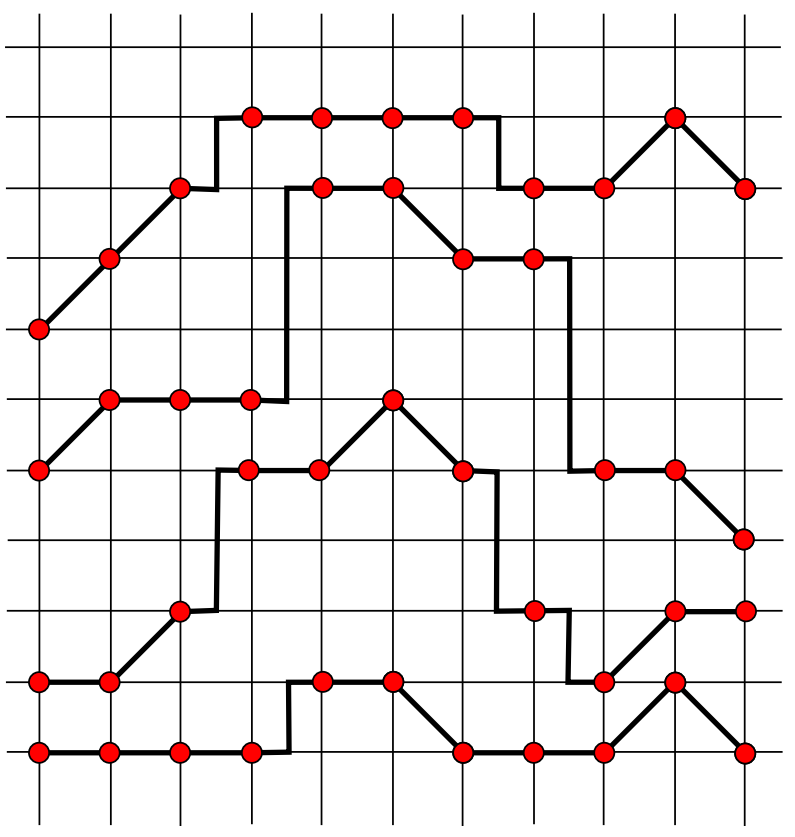

Figure 1: The first two columns, together with the 5-th and the 9-th display ascending $\beta$-paths, the 3 -rd and the 4 -th - ascending $\alpha$-paths, the 6 -th and the 10 -th - the descending $\beta$-paths, the 7 -th and the 8 -th - the descending $\alpha$-paths.

the three-link path

$$
\begin{gathered}
\omega_{x, \pi^{+}(x)}=\left[(k, x),\left(k+\frac{1}{2}, x\right)\right] \cup\left[\left(k+\frac{1}{2}, x\right),\left(k+\frac{1}{2}, \pi^{+}(x)\right)\right] \\
\cup\left[\left(k+\frac{1}{2}, \pi^{+}(x)\right),\left(k+1, \pi^{+}(x)\right)\right] .
\end{gathered}
$$

Then for different particles $n_{\left(k, x^{\prime}\right)}=1, n_{\left(k, x^{\prime \prime}\right)}=1$ the connectors $\omega_{x^{\prime}, \pi^{+}\left(x^{\prime}\right)}$, $\omega_{x^{\prime \prime}, \pi^{+}\left(x^{\prime \prime}\right)}$ do not intersect. We define the action $S$ on each of these connectors by

$$
S\left(\omega_{x, \pi^{+}(x)}\right)=\left(\pi^{+}(x)-x\right) \ln \alpha_{k}^{+} .
$$

In the case when $\psi_{k}(u)=\left(1-\alpha_{k}^{-} u^{-1}\right)^{-1}$ the situation is very similar, except the upward interlacing is replaced by the downward interlacing: The correspondence $\pi^{+}$is replaced by $\pi^{-}$, which assigns to a particle $n_{(k, x)}=1$ the particle $n_{\left(k+1, \pi^{-}(x)\right)}=1$, where $\pi^{-}(x)=\max \left\{y \leq x: n_{(k+1, y)}=1\right\} \leq x$. 
Again, $\pi^{-}$is one-to-one a.s., and the (downward) connectors

$$
\begin{gathered}
\omega_{x, \pi^{-}(x)}=\left[(k, x),\left(k+\frac{1}{2}, x\right)\right] \cup\left[\left(k+\frac{1}{2}, x\right),\left(k+\frac{1}{2}, \pi^{-}(x)\right)\right] \\
\cup\left[\left(k+\frac{1}{2}, \pi^{-}(x)\right),\left(k+1, \pi^{-}(x)\right)\right]
\end{gathered}
$$

do not intersect. The action $S$ is given by

$$
S\left(\omega_{x, \pi^{-}(x)}\right)=\left(x-\pi^{-}(x)\right) \ln \alpha_{k}^{-} .
$$

For $\psi_{k}(u)=\left(1+\beta_{k}^{+} u\right)$ we have the upward interlacing of holes: If in the configuration $n$ we have two holes $n_{\left(k, x_{1}\right)}=n_{\left(k, x_{2}\right)}=0, x_{1}<x_{2}$, separated by the string of particles $\left\{n_{(k, x)}=1\right.$ for all $\left.x_{1}<x<x_{2}\right\}$, then on the neighboring line $\sigma=k+1$ we have $\mathbf{P r}_{K}$-a.s. exactly one hole $n_{(k+1, x)}=0$ in the set $\left\{(k+1, x): x_{1}<x \leq x_{2}\right\}$, while the rest of points in this set is filled by the particles. We then define a correspondence $\chi^{+}$, assigning to every particle on the $\sigma=k$ line a particle on the $\sigma=k+1$ line, as follows. Take any string of consecutive particles $\left\{n_{(k, x)}=1\right.$ for all $\left.x_{1}<x<x_{2}\right\}$ which is maximal, i.e. $n_{\left(k, x_{1}\right)}=n_{\left(k, x_{2}\right)}=0$. We put

$$
\chi^{+}\left(x_{1}+1\right)=\min \left\{x \geq x_{1}+1: n_{(k+1, x)}=1\right\},
$$

and then proceed inductively by putting

$$
\chi^{+}(x+1)=\min \left\{y>\chi^{+}(x): n_{(k+1, y)}=1\right\} .
$$

The hole interlacing implies that $\chi^{+}$is a.s. well-defined, is one-to-one, and that for every particle $n_{(k, x)}=1$ we have either $\chi^{+}(x)=x$ or $\chi^{+}(x)=x+1$. The particle connectors, which in this case are segments

$$
\omega_{x, \chi^{+}(x)}=\left[(k, x),\left(k+1, \chi^{+}(x)\right)\right],
$$

clearly do not intersect each other. We put

$$
S\left(\omega_{x, \chi^{+}(x)}\right)=\left\{\begin{array}{cc}
0 & \text { if } \chi^{+}(x)=x, \\
\ln \beta_{k}^{+} & \text {if } \chi^{+}(x)=x+1 .
\end{array}\right.
$$

For $\psi_{k}(u)=\left(1+\beta_{k}^{-} u^{-1}\right)$ we have likewise the downward interlacing of holes. The correspondence $\chi^{+}$is replaced by $\chi^{-}$, with the property that either $\chi^{-}(x)=x$ or $\chi^{-}(x)=x-1$. The connectors are non-intersecting segments

and we define

$$
\omega_{x, \chi^{-}(x)}=\left[(k, x),\left(k+1, \chi^{-}(x)\right)\right],
$$

$$
S\left(\omega_{x, \chi^{-}(x)}\right)=\left\{\begin{array}{cc}
0 & \text { if } \chi^{-}(x)=x \\
\ln \beta_{k}^{-} & \text {if } \chi^{-}(x)=x-1 .
\end{array}\right.
$$




\subsection{Main result}

Now we can formulate our claims more precisely. Let us fix a sequence of functions $\psi_{k}(u), k \in \mathbb{Z}^{1}$, such that for every $k$ the function $\psi_{k}(u)$ is one of the four functions $\left(1-\alpha_{k}^{+} u\right)^{-1},\left(1-\alpha_{k}^{-} u^{-1}\right)^{-1},\left(1+\beta_{k}^{+} u\right),\left(1+\beta_{k}^{-} u^{-1}\right)$, with $\alpha_{*}^{ \pm}, \beta_{*}^{ \pm}>0$. Let us also fix a complex number $z, \Im z>0$.

Theorem 1 i) The kernel (2) defines a determinantal random field $n$ on $\mathbb{Z}^{2}$, which is invariant with respect to the shifts of the second coordinate.

ii) The random field $n$ possesses the interlacing property as defined in Section 1.2. In particular, there is a map $\omega$, assigning to $\mathbf{P r}_{K}$-a.e. realization of $n$ a countable collection of non-intersecting lattice paths $\omega_{n}=\left\{\omega_{i}\right\}$, passing through all the particles of the configuration $n$. The construction of the collection $\omega_{n}$ is given by (4), (6) , (18) and (10).

iii) The random paths $\omega$ thus constructed form Gibbs Path Ensemble, as defined in Section 1.1. It corresponds to the action functional S, given by the formulas (5), (7), (9) and (11).

iv) For every $k \in \mathbb{Z}$ the matrix elements of the kernel $K$ satisfy the following relations:

for the case $\psi_{k}(u)=\left(1-\alpha_{k}^{+} u\right)^{-1}$

$$
\begin{aligned}
& K_{k-1, \tau}(x-y)-\delta_{\substack{x=y-1 \\
\tau=k-1}}=K_{k, \tau}(x-y)-\alpha_{k}^{+} K_{k, \tau}(x-y-1) \text {, } \\
& K_{\sigma, k}(x-y)-\delta_{\substack{x=y \\
\sigma=k}}=K_{\sigma, k-1}(x-y)-\alpha_{k}^{+} K_{\sigma, k-1}(x-y-1) ;
\end{aligned}
$$

for the case $\psi_{k}(u)=\left(1+\beta_{k}^{+} u\right)$

$$
\begin{aligned}
& K_{k, \tau}(x-y)=\left[K_{k-1, \tau}(x-y)-\delta_{\substack{x=y \\
\tau=k-1}}\right]+\beta_{k}^{+}\left[K_{k-1, \tau}(x-y-1)-\delta_{x=y+1}\right], \\
& K_{\sigma, k-1}(x-y)=\beta_{k}^{+}\left[K_{\sigma, k}(x-y-1)-\delta_{\substack{\sigma=k \\
x=y+1}}\right]+\left[K_{\sigma, k}(x-y)-\delta_{\sigma=k} x\right] \text {; }
\end{aligned}
$$

and similar relations for the $\alpha^{-}, \beta^{-}$cases. The determinant identities, expressing the properties $\mathbf{i i )}$ and $\mathbf{i i i}$ ) above, are corollaries of these relations only, and thus hold true for any other kernel $K$, satisfying them. 


\section{Examples}

1. Our first example will be Gibbs ensembles of the $\beta$-paths, introduced in (8) , (10) . These are collections of non-intersecting infinite paths $\left\{\omega_{i}\right\}$ on $\mathbb{Z}^{2}$, such that if a path visits the point $(\sigma, x)$, then its next link is either the segment $[(\sigma, x),(\sigma+1, x)]$ or the segment $[(\sigma, x),(\sigma+1, x+1)]$. Now let $\varrho=\varrho(p, q)$ be a finite piece of such $\beta$-path, where $p, q$ are the end-points of $\varrho$. We define the energy $U(\varrho)$ of this path in the following way. Let $\varrho_{-}(p, q)$ be the $\beta$-path, which is the lowest among all the $\beta$-paths connecting $p$ and $q$. Then $\exp \{-U(\varrho)\}$ is by definition the area surrounded by the loop $\varrho(p, q) \cup \varrho_{-}(p, q)$. For a collection $\varrho=\left\{\varrho_{i}\right\}$ of finite paths we define $H(\varrho)=\sum_{i} U\left(\varrho_{i}\right)$.

We call the measure $\mu$ on the ensemble $\omega$ of non-intersecting infinite $\beta$ paths the Gibbs measure corresponding to the energy $H$ and the inverse temperature $\tau$, if it has the following property. Let $\Lambda \subset \mathbb{Z}^{2}$ be a finite volume, and the sets $P=\left\{p_{1}, \ldots, p_{k} \in \partial \Lambda\right\}, Q=\left\{q_{1}, \ldots, q_{k} \in \partial \Lambda\right\}$ of the entrance points and exit points are fixed. Then the conditional distribution of $\mu$ on $\Omega_{\Lambda}(P, Q)=\left\{\varrho_{\Lambda}\right\}$ under the condition that the path configuration $\omega_{\bar{\Lambda}}$ is fixed outside $\Lambda$ is given by the formula

$$
\mu\left(\varrho_{\Lambda} \mid \omega_{\bar{\Lambda}}\right)=\frac{\exp \left\{-\tau H\left(\varrho_{\Lambda}\right)\right\}}{\bar{Z}(\Lambda, P, Q)}
$$

This definition is just a convenient rewriting of the one given above. The advantage is that our function $H$ here is manifestly translation-invariant. Our main result implies in particular that the determinantal random fields $\mu_{\varkappa, z}$ defined by the kernel $K=K(\varkappa, z)$ with the functions $\psi_{k}(u)=\left(1+\varkappa e^{k \tau} u\right)$, interpreted as path measures, are Gibbs measures with the energy $H$ and the inverse temperature $\tau$. Here $\varkappa>0$ is any real number.

When the temperature $\tau^{-1}$ goes to zero, the Gibbs measures (12) are concentrated on ground-state configurations, which are local minima of the energy $H$. For low temperatures they are concentrated on configurations which

are small perturbations of the ground state configurations, see Fig. 2. Note that the ground state configurations have their corner points confined to at most two nearest neighbor vertical lattice lines. One can say that for large $\tau$ our two-dimensional random field is essentially one-dimensional, and outside the strip of width $\sim \tau^{-1}$ it is basically frozen. Along this vertical direction it has the following correlation decay property: for every two finite 
subsets $A, B \subset \mathbb{Z}^{2}$ we have $\left\langle n_{A+x} n_{B}\right\rangle-\left\langle n_{A}\right\rangle\left\langle n_{B}\right\rangle \rightarrow 0$ as $|x| \rightarrow \infty$. Here $n_{A}=\prod_{(\sigma, x) \in A} n_{\sigma, x}$.
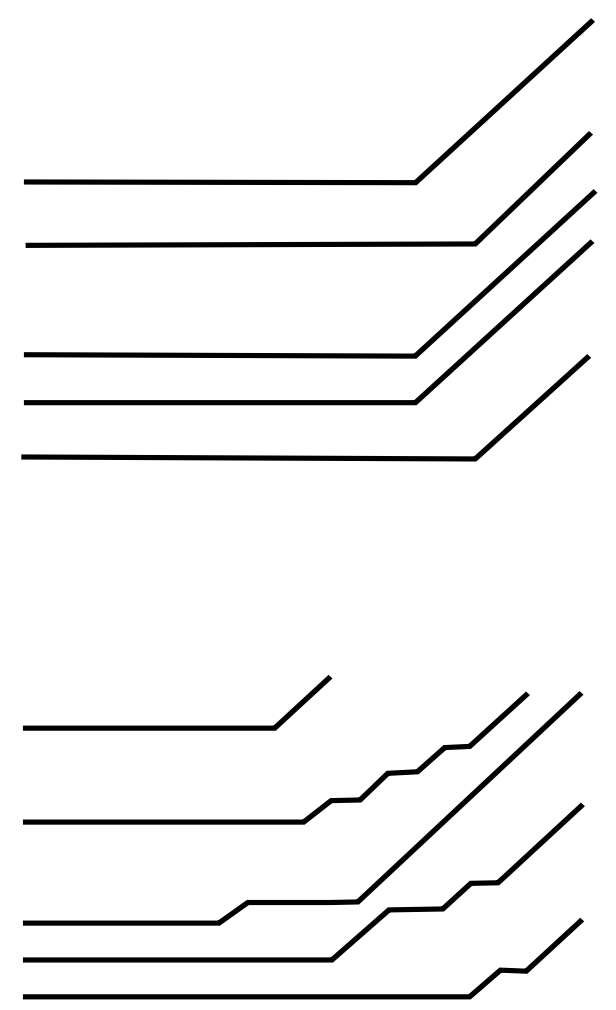

Figure 2: A ground state configuration of the $\beta$-paths, and a low-temperature configuration.

Without loss of generality we can assume that $|z|=1$. The parameter $z$ then defines the "slope" of the "height function", or, what is the same, the (constant) density of the paths in the path ensemble.

Note now, that for every $z$ the determinantal processes $\mu_{\varkappa, z}$ are different for different values of $\varkappa$. This follows just from the computation of the second correlation function for these processes. That means that there are continuum non-translation-invariant Gibbs measures (12), corresponding to the same slope and the same temperature. Of course, the field $\mu_{\varkappa, z}$ is just a translate of the field $\mu_{\tau \varkappa, z}$ by the unit lattice vector. But the fields $\mu_{\varkappa, z}$ with $\varkappa$ between 1 and $\tau$ are all different and are not related by the lattice shift transformation. 


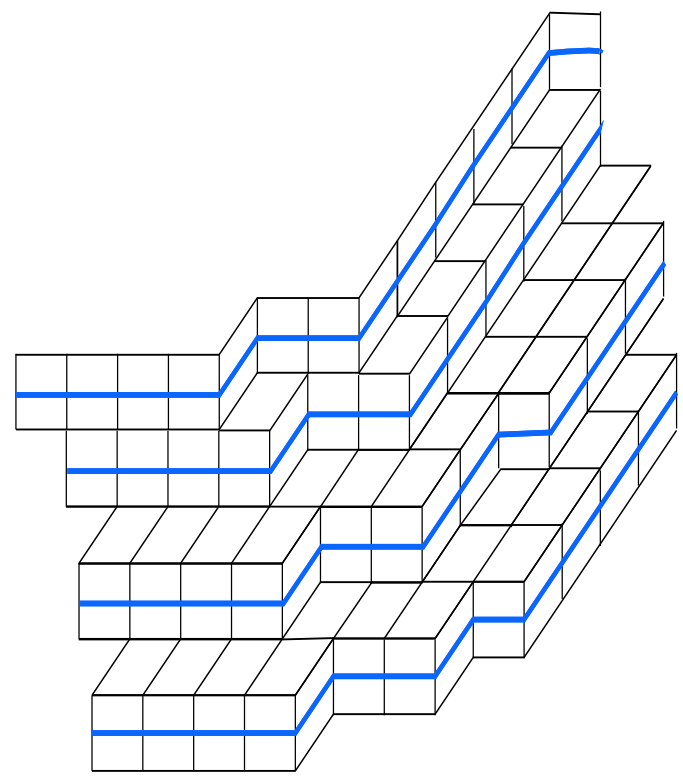

Figure 3: The $\beta$-paths and the corresponding (deformed-)lozenge tiling.

One can understand better the role played by the parameter $\varkappa$ looking at the boundary conditions and the limiting behavior of the processes $\mu_{\varkappa, z}$. The restriction of the process to any vertical line $\sigma=$ const gives the (translationinvariant) sine process with density $\arg z / \pi$. Let us consider two such lines, say $\sigma= \pm k$. Then the paths of the process $\mu_{\varkappa, z}$ define in a natural way the coupling $\mathcal{C}(k, \varkappa, \tau)$ between the two sine processes. Consider the limiting coupling $\mathcal{C}(\varkappa, \tau)=\lim _{k \rightarrow \infty} \mathcal{C}(k, \varkappa, \tau)$. It turns out that the couplings $\mathcal{C}(\varkappa, \tau)$ are still non-trivial (i.e. these couplings are not product-couplings), and are different for different $\varkappa$. The two couplings $\mathcal{C}(\varkappa, \tau)$ and $\mathcal{C}(\tau \varkappa, \tau)$ are related by the unit shift of one of the sin-processes. The couplings $\mathcal{C}(\varkappa, \tau)$ become trivial only in the limit when $\tau \rightarrow 0$. In this limit the fields $\mu_{\varkappa, z}$ become fully translation invariant.

A straightforward geometric interpretation of our ensemble of the $\beta$-paths is to relate them to the lozenge tilings of the plane. Our paths are then composed by the middle lines of all "vertical" plaquettes, see Fig. 3, cf. [8].

One may wonder about the existence of the asymptotic shape of the height function corresponding to the field $\mu_{1, z}$, i.e. to the choice $\psi_{k}(u)=\left(1+\tau^{k} u\right)$. However, this height function is almost frozen outside the strip of width 


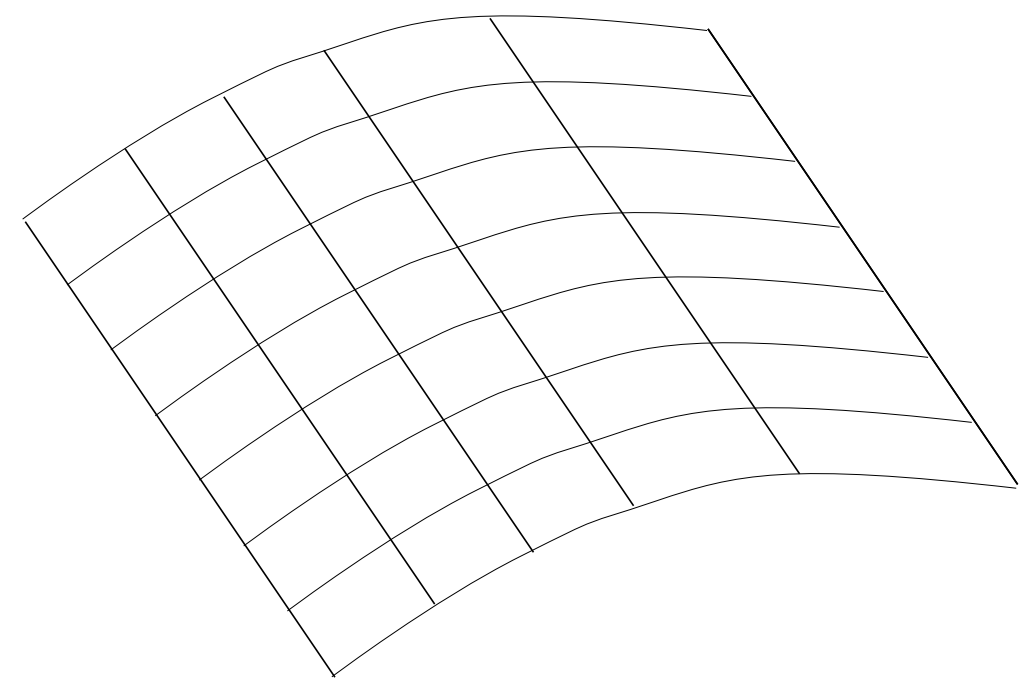

Figure 4: The limit shape

$\sim \tau^{-1}$ around the line $\sigma=0$. If we scale this surface by the factor $\tau$ in all three dimensions, then the conjectural limit when $\tau \rightarrow 0$ is a non-random cylindrical surface. This surface has a gutter shape, see Fig. 4, and is given by the following geometric construction.

To describe it we first recall the geometric construction (see [15] or [16]), used to obtain the limit shape $\mathcal{C}_{C K}$ of the plane partitions by Cerf and Kenyon in [5]. For every positive unit vector $\mathbf{n} \in \Delta^{2}=S^{2} \cap \mathbb{R}_{+}^{3}$ let ent (n) be the residual entropy of the lozenge tilings having the slope plane orthogonal to $\mathbf{n}$ (see Theorem 1.1 in [5]). Now define the halfspaces

$$
K_{\mathbf{n}}=\left\{\mathbf{x} \in \mathbb{R}^{3}:(\mathbf{x}, \mathbf{n}) \geq \operatorname{ent}(\mathbf{n})\right\} .
$$

Let

$$
K=\cap_{\mathbf{n} \in \Delta^{2}} K_{\mathbf{n}}
$$

the boundary of the region $K$ is precisely the surface $\mathcal{C}_{C K}$, describing the typical shape of a large plane partition.

To describe the gutter shape we first define its slope, $\gamma$. This is determined by the frequency of the lines in our family (indeed, they are just the level lines of the height function). If $z=e^{i \varphi}$, then the density in question is the first correlation function of our determinantal process, and it is equal to $\frac{\varphi}{\pi}$. Therefore $\gamma$ satisfies $\tan \gamma=\frac{\varphi}{1-\varphi}$. Let us define the vector 
$\mathbf{m}(\gamma)=\left(m_{x}, m_{y}, m_{z}\right)=\left(-\frac{1}{\sqrt{2}},-\frac{1}{\sqrt{2}}, \tan \gamma\right)$. This is the direction of our gutter. Consider the arc $A(\gamma)$ of vectors in the "triangle" $\Delta^{2}$, which are orthogonal to $\mathbf{m}(\gamma)$ :

$$
A(\gamma)=\left\{\mathbf{n} \in \Delta^{2}:(\mathbf{n}, \mathbf{m}(\gamma))=0\right\} .
$$

Our gutter surface, $G(\gamma)$, is defined to be the boundary of the convex region

$$
K(\gamma) \equiv \cap_{\mathbf{n} \in A(\gamma)} K_{\mathbf{n}}
$$

Note that the surface $G(\gamma)$ consists of straight lines parallel to the vector $\mathbf{m}(\gamma)$. The surfaces $G(\gamma)$ and $\mathcal{C}_{C K}$ are tangent to each other along the common curve $g(\gamma)=G(\gamma) \cap \mathcal{C}_{C K}$. Each of the curves $g(\gamma)$ is a smooth curve without straight pieces. Asymptotically each of them approaches the Vershik curve $\mathcal{C}_{V}:\left\{\exp \left(-\frac{\pi}{\sqrt{6}} x\right)+\exp \left(-\frac{\pi}{\sqrt{6}} y\right)=1, z=0\right\}$, which belongs to the boundary of the curved part of the surface $\mathcal{C}_{C K}$.

2. Our second example is the ensemble of $\alpha \beta$-paths, with $\psi_{2 k}(u)=$ $\left(1-\alpha_{k}^{-} u^{-1}\right)^{-1}$ and $\psi_{2 k+1}(u)=\left(1+\beta_{k}^{+} u\right)$. Again we will choose $\alpha$ and $\beta$ to be geometric progressions, by putting $\alpha_{k}^{-}=\left(\varkappa e^{k \tau}\right)^{-1}, \beta_{k}^{+}=\lambda e^{k \tau}$, with $\varkappa, \lambda, \tau>0$. In the same way that the $\beta$-paths are related to the lozenge tiling, the $\alpha \beta$-paths are related to the domino tilings. The relation however is not so easy to explain; the corresponding construction, establishing the bijection between the two entities, is presented in [9], see also [12].

The Fig. 5 shows one collection of $\alpha \beta$-paths with $\varkappa=\lambda=1$ and $\tau=\infty$ (which therefore should be called a ground state configuration).

For $k>0$ all the $\alpha$-steps are zero height steps, while all the $\beta$-steps are ascending. For $k<0$ all the $\beta$-steps are zero height steps, while all the $\alpha$-steps are descending in a maximal possible way. The Fig. 6 shows the corresponding domino tiling.

In domino tilings, the elementary moves correspond to finding a $2 \times$ 2 square tiled by two dominoes, say horizontal, and replacing this pair of dominos by two vertical ones. If one assigns four weights $a, b, c, d$ to the four possible positions of a single domino, then every move replacing a horizontal pair by a vertical one changes the overall weight by a constant factor. (If $a, b$ are two horizontal weights, then the overall change will be by a factor $\frac{c d}{a b}$.) In our case the overall weight after an elementary move depends on the parity of the $2 \times 2$ square, and is $\frac{\varkappa}{\lambda}$ in one case, and $\frac{\varkappa}{\lambda} e^{\tau}$ in the other. This means that the measures on the domino tilings that we have constructed, can not be obtained by assigning weights to single dominoes. 


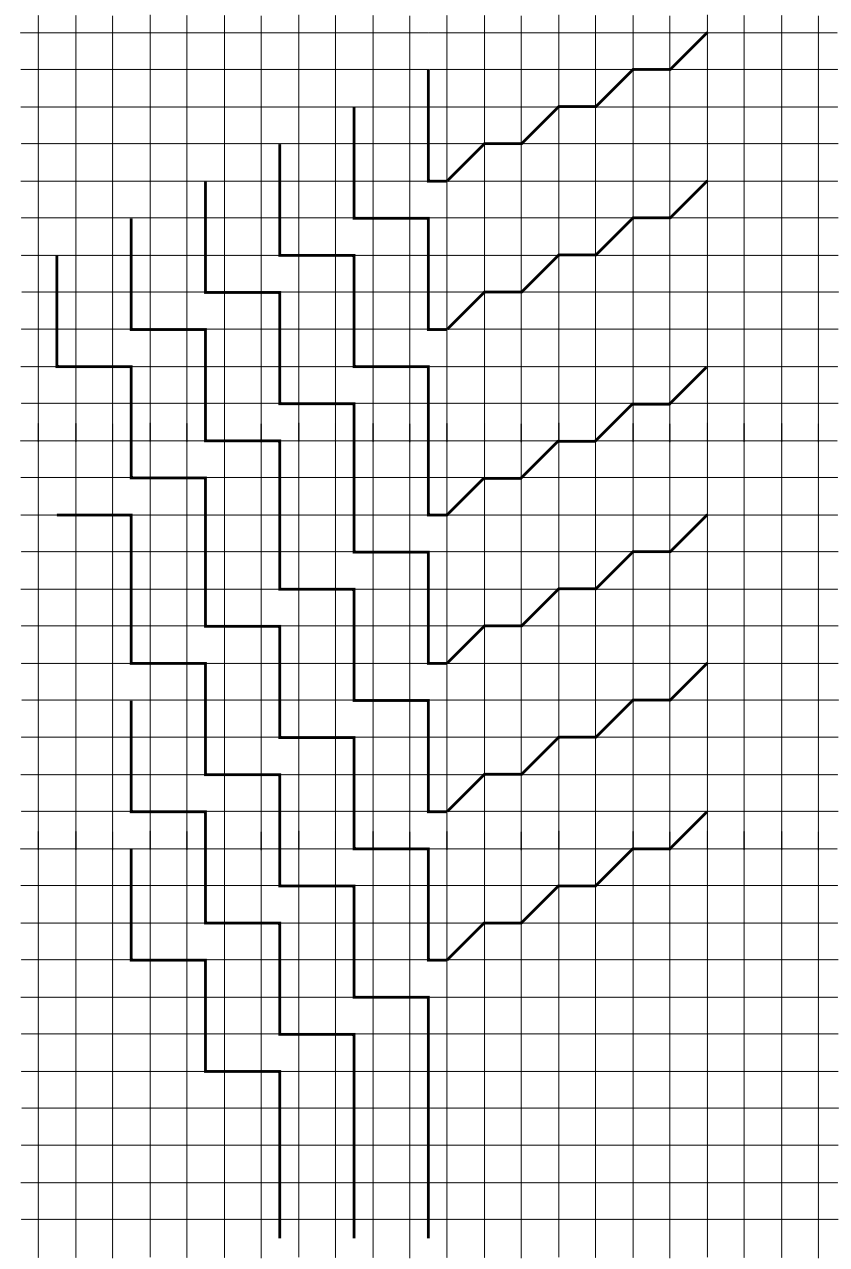

Figure 5: A ground state configuration of $\alpha \beta$-paths. 


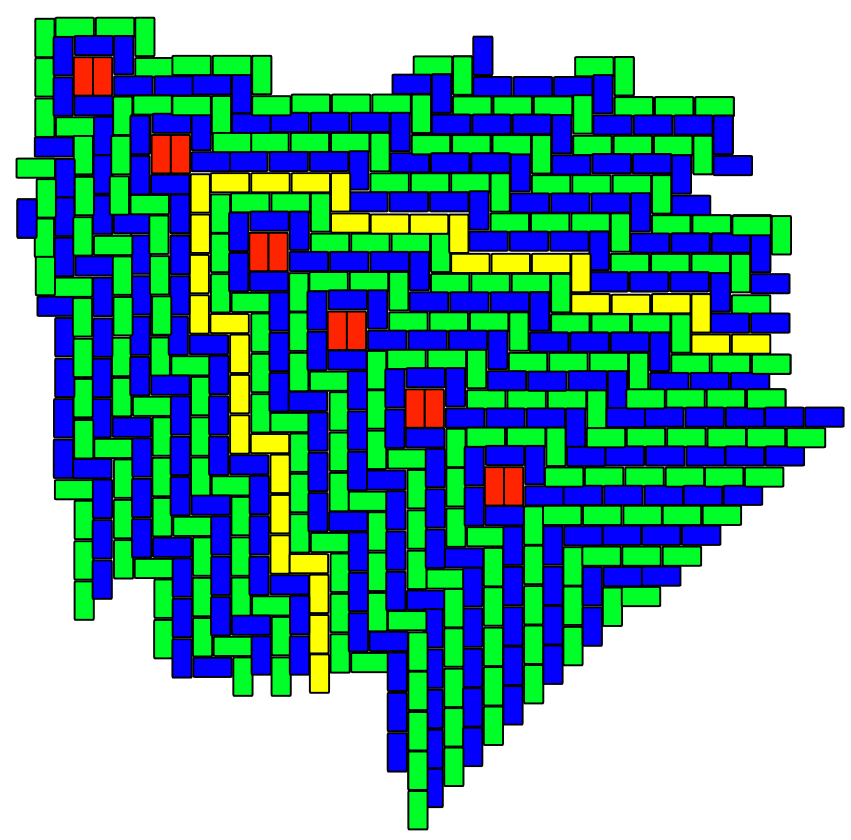

Figure 6: The domino tiling, corresponding to the paths above. 
Again, for $\tau \neq 0$ our measures are non-translation-invariant, and by varying the ratio $\frac{\varkappa}{\lambda}$ we obtain a whole continuum of different measures.

In the case $\tau=0$, the two-parametric measure on lozenge tilings and the three-parametric measure on domino tilings are translation invariant with respect to all shifts of $\mathbb{Z}^{2}$. These measures are well known; for lozenge tilings they were obtained in [10, [13], and for domino tilings they were obtained in [4, 6], see also 9]. As proved in [14, they are the only fully translation invariant ergodic measures.

\section{Proof of the Main Result}

The proof of $\mathbf{i}$ of Theorem 1 is given in Section 4 below.

We will start the proof of ii-iv by dealing with the special case when for each $k \in \mathbb{Z}^{1}$ the function $\psi_{k}(u)$ is either $\left(1-\alpha_{k} u\right)^{-1}$ or $\left(1+\beta_{k} u\right)$, with $\alpha_{k}, \beta_{k}$ some positive constants. We will consider the general case at the end of the proof.

\subsection{Linear relations}

Let the sequence of functions $\psi_{k}(u)$ be given, where for every $k \in \mathbb{Z}^{1}$ the function $\psi_{k}(u)$ is either $\left(1-\alpha_{k} u\right)^{-1}$ or $\left(1+\beta_{k} u\right)$, with $\alpha_{k}, \beta_{k}$ some positive constants.

In this subsection we will show that the kernel $K_{\sigma, \tau}(x-y)$ satisfies the linear relations mentioned in Theorem 1. Indeed, if $\psi_{k}(u)=\left(1-\alpha_{k} u\right)^{-1}$, then for $\sigma=k-1$ and $\tau \geq k$

$$
\begin{aligned}
K_{k-1, \tau}(x-y) & =\frac{1}{2 \pi i} \int_{C_{+}}\left(1-\alpha_{k} u\right)\left(\prod_{k+1}^{\tau} \psi_{j}(u)\right)^{-1} \frac{d u}{u^{x-y+1}} \\
& =K_{k, \tau}(x-y)-\alpha_{k} K_{k, \tau}(x-y-1)
\end{aligned}
$$


the same for $\tau<k-1$ :

$$
\begin{aligned}
& K_{k, \tau}(x-y)-\alpha_{k} K_{k, \tau}(x-y-1) \\
& =\frac{1}{2 \pi i} \int_{C_{-}} \prod_{\tau+1}^{k-1} \psi_{j}(u)\left(1-\alpha_{k} u\right)^{-1} \frac{d u}{u^{x-y+1}}-\alpha_{k} \frac{1}{2 \pi i} \int_{C_{-}} \prod_{\tau+1}^{k-1} \psi_{j}(u)\left(1-\alpha_{k} u\right)^{-1} \frac{d u}{u^{x-y}} \\
& =\frac{1}{2 \pi i} \int_{C_{-}} \prod_{\tau+1}^{k-1} \psi_{j}(u) \frac{d u}{u^{x-y+1}}=K_{k-1, \tau}(x-y)
\end{aligned}
$$

while for $\tau=k-1$ we have

$$
\begin{aligned}
& K_{k-1, k-1}(x-y)=\frac{1}{2 \pi i} \int_{C_{+}} \frac{d u}{u^{x-y+1}} \\
& K_{k, k-1}(x-y)-\alpha_{k} K_{k, k-1}(x-y-1) \\
&=\frac{1}{2 \pi i} \int_{C_{-}}\left(1-\alpha_{k} u\right)^{-1} \frac{d u}{u^{x-y+1}}-\alpha_{k} \frac{1}{2 \pi i} \int_{C_{-}}\left(1-\alpha_{k} u\right)^{-1} \frac{d u}{u^{x-y}} \\
&=\frac{1}{2 \pi i} \int_{C_{-}} \frac{d u}{u^{x-y+1}},
\end{aligned}
$$

which means that

$$
K_{k-1, k-1}(x-y)-K_{k, k-1}(x-y)+\alpha_{k} K_{k, k-1}(x-y-1)=\delta_{x=y} .
$$

Altogether, these relations read

$$
K_{k-1, \tau}(x-y)-\delta_{\tau=k-1}^{x=y}=K_{k, \tau}(x-y)-\alpha_{k} K_{k, \tau}(x-y-1) .
$$

Also, if $\tau=k$ and $\sigma \leq k-1$, then

$$
\begin{aligned}
K_{\sigma, k}(x-y) & =\frac{1}{2 \pi i} \int_{C_{+}}\left(\prod_{\sigma+1}^{\tau} \psi_{j}(u)\right)^{-1}\left(1-\alpha_{k} u\right) \frac{d u}{u^{x-y+1}} \\
& =K_{\sigma, k-1}(x-y)-\alpha_{k} K_{\sigma, k-1}(x-y-1)
\end{aligned}
$$

and the same for $\sigma>k$. Since the diagonal elements $K_{r, r}(x-y)$ do not depend on $r$, for $\sigma=k$ we have immediately from (13) :

$$
K_{k, k}(x-y)-K_{k, k-1}(x-y)+\alpha_{k} K_{k, k-1}(x-y-1)=\delta_{x=y} .
$$


Altogether,

$$
K_{\sigma, k}(x-y)-\underset{\substack{x=y \\ \sigma=k}}{\delta}=K_{\sigma, k-1}(x-y)-\alpha_{k} K_{\sigma, k-1}(x-y-1) .
$$

Likewise, for $\psi_{k}(u)=\left(1+\beta_{k} u\right), \sigma=k$ and $k-1>\tau$ we have

$$
\begin{aligned}
K_{k, \tau}(x-y) & =\frac{1}{2 \pi i} \int_{C_{-}}\left(\prod_{\tau+1}^{k-1} \psi_{j}(u)\right)\left(1+\beta_{k} u\right) \frac{d u}{u^{x-y+1}} \\
& =K_{k-1, \tau}(x-y)+\beta_{k} K_{k-1, \tau}(x-y-1)
\end{aligned}
$$

and similarly for $k-1<\tau$. For $\tau=k-1$ we have

$$
K_{k, k-1}(x-y)=\frac{1}{2 \pi i} \int_{C_{-}}\left(1+\beta_{k} u\right) \frac{d u}{u^{x-y+1}}
$$

while

$K_{k-1, k-1}(x-y)+\beta_{k} K_{k-1, k-1}(x-y-1)=\frac{1}{2 \pi i} \int_{C_{+}} \frac{d u}{u^{x-y+1}}+\beta_{k} \frac{1}{2 \pi i} \int_{C_{+}} \frac{d u}{u^{x-y}}$,

SO

$$
\begin{aligned}
& K_{k-1, k-1}(x-y)+\beta_{k} K_{k-1, k-1}(x-y-1)-K_{k, k-1}(x-y) \\
& =\frac{1}{2 \pi i} \oint\left(1+\beta_{k} u\right) \frac{d u}{u^{x-y+1}}=\delta_{x=y}+\beta_{k} \delta_{x=y+1} .
\end{aligned}
$$

Summarizing, we have

$$
K_{k, \tau}(x-y)=\left[K_{k-1, \tau}(x-y)-\delta_{\substack{x=y \\
\tau=k-1}}\right]+\beta_{k}\left[K_{k-1, \tau}(x-y-1)-\begin{array}{r}
\delta_{x=y+1} \\
\tau=k-1
\end{array}\right] .
$$

The last relation we obtain by considering for $\psi_{k}(u)=\left(1+\beta_{k} u\right)$ the case when $\sigma>k$ while $\tau=k-1$. Then we have

$$
\begin{aligned}
K_{\sigma, k-1}(x-y) & =\frac{1}{2 \pi i} \int_{C_{-}}\left(1+\beta_{k} u\right)\left(\prod_{k+1}^{\sigma} \psi_{j}(u)\right) \frac{d u}{u^{x-y+1}} \\
& =K_{\sigma, k}(x-y)+\beta_{k} K_{\sigma, k}(x-y-1) .
\end{aligned}
$$

The same relation holds in the region $\sigma<k$, while for $\sigma=k$ we use (18), which immediately imply that

$$
K_{k, k}(x-y)+\beta_{k} K_{k, k}(x-y-1)-K_{k, k-1}(x-y)=\delta_{x=y}+\beta_{k} \delta_{x=y+1},
$$


thus getting us to

$$
K_{\sigma, k-1}(x-y)=\beta_{k}\left[K_{\sigma, k}(x-y-1)-\delta_{\substack{\sigma=k \\ x=y+1}}\right]+\left[K_{\sigma, k}(x-y)-\delta_{\sigma=k} x .\right.
$$

\subsection{Interlacing property. Simplest case.}

Let us start by checking the interlacing property in the simplest situations. In the case $\psi_{k}(u)=\left(1-\alpha_{k} u\right)^{-1}$ we will show that

$$
\operatorname{Pr}_{K}\left\{\left(\begin{array}{cc}
1 & * \\
1 & 0
\end{array}\right)_{k-1, k}\right\}=0, \quad \operatorname{Pr}_{K}\left\{\left(\begin{array}{ll}
0 & 1 \\
* & 1
\end{array}\right)_{k-1, k}\right\}=0
$$

where the symbol $\left(\begin{array}{ll}1 & * \\ 1 & 0\end{array}\right)_{k-1, k}$ denotes the corresponding event in some two by two square in the vertical strip $\{(k-1, *),(k, *)\}$. For the case $\sigma=k$ with $\psi_{k}(u)=\left(1+\beta_{k} u\right)$ we will show that

$$
\operatorname{Pr}_{K}\left\{\left(\begin{array}{ll}
0 & 1 \\
0 & *
\end{array}\right)_{k-1, k}\right\}=0, \quad \operatorname{Pr}_{K}\left\{\left(\begin{array}{cc}
* & 0 \\
1 & 0
\end{array}\right)_{k-1, k}\right\}=0 .
$$

To save on notation, we will put $k=1$, and we will write $\alpha, \beta, \psi$ instead of $\alpha_{1}, \beta_{1}, \psi_{1}$.

1. The case $\psi(u)=(1-\alpha u)^{-1}$ :

$$
\operatorname{Pr}_{K}\left\{\left(\begin{array}{ll}
1 & * \\
1 & 0
\end{array}\right)_{0,1}\right\}=0
$$

This relation is equivalent to showing that

$$
\operatorname{det}\left|\begin{array}{ccc}
K_{0,0}(0) & K_{0,0}(-1) & K_{0,1}(0) \\
K_{0,0}(1) & K_{0,0}(0) & K_{0,1}(1) \\
K_{1,0}(0) & K_{1,0}(-1) & K_{1,1}(0)-1
\end{array}\right|=0 .
$$

But this relation does hold, since the relation (15) implies that the last column is a linear combination of the remaining two. 
2. The case $\psi(u)=(1-\alpha u)^{-1}$ :

$$
\operatorname{Pr}_{K}\left\{\left(\begin{array}{ll}
0 & 1 \\
* & 1
\end{array}\right)_{0,1}\right\}=0
$$

We have to check that

$$
\operatorname{det}\left|\begin{array}{ccc}
K_{1,1}(0) & K_{1,1}(-1) & K_{1,0}(-1) \\
K_{1,1}(1) & K_{1,1}(0) & K_{1,0}(0) \\
K_{0,1}(1) & K_{0,1}(0) & K_{0,0}(0)-1
\end{array}\right|=0 .
$$

But from (14) it follows that the last row is a combination of the remaining two.

3. The case $\psi(u)=(1+\beta u)$ :

$$
\operatorname{Pr}_{K}\left\{\left(\begin{array}{ll}
0 & 1 \\
0 & *
\end{array}\right)_{0,1}\right\}=0
$$

We have thus to show the vanishing of

$$
\operatorname{det}\left|\begin{array}{ccc}
K_{0,0}(0)-1 & K_{0,0}(-1) & K_{0,1}(-1) \\
K_{0,0}(1) & K_{0,0}(0)-1 & K_{0,1}(0) \\
K_{1,0}(1) & K_{1,0}(0) & K_{1,1}(0)
\end{array}\right| .
$$

But the relation (20) tells us that the third row of the last determinant is a linear combination of the first two.

4. The case $\psi(u)=(1+\beta u)$ :

$$
\operatorname{Pr}_{K}\left\{\left(\begin{array}{ll}
* & 0 \\
1 & 0
\end{array}\right)_{0,1}\right\}=0
$$

We thus need the vanishing of the determinant

$$
\operatorname{det}\left|\begin{array}{ccc}
K_{0,0}(0) & K_{0,1}(0) & K_{0,1}(-1) \\
K_{1,0}(0) & K_{1,1}(0)-1 & K_{1,1}(-1) \\
K_{1,0}(1) & K_{1,1}(1) & K_{1,1}(0)-1
\end{array}\right| .
$$

But the first column is a combination of the second and the third, due to (21) . 


\subsection{Elementary moves.}

Here we will prove another set of identities, corresponding to the elementary moves of the paths. Since every move involves two adjacent columns of the lattice, we have four different types of moves, according to the four types $\alpha \alpha, \alpha \beta, \beta \alpha$, or $\beta \beta$ - of the columns pair.

1. We start with the case $\psi_{1}(u)=\left(1+\beta_{1} u\right), \psi_{2}(u)=\left(1+\beta_{2} u\right)$. We will prove that

$$
\beta_{1} \operatorname{Pr}_{K}\left\{\left(\begin{array}{ccc}
* & 0 & 1 \\
1 & 1 & *
\end{array}\right)_{0,1,2}\right\}=\beta_{2} \operatorname{Pr}_{K}\left\{\left(\begin{array}{ccc}
* & 1 & 1 \\
1 & 0 & *
\end{array}\right)_{0,1,2}\right\} .
$$

The corresponding determinant relation reads:

$$
\begin{aligned}
& \beta_{1} \operatorname{det}\left|\begin{array}{cccc}
K_{0,0}(0) & K_{0,1}(0) & K_{0,1}(-1) & K_{0,2}(-1) \\
K_{1,0}(0) & K_{1,1}(0) & K_{1,1}(-1) & K_{1,2}(-1) \\
K_{1,0}(1) & K_{1,1}(1) & K_{1,1}(0)-1 & K_{1,2}(0) \\
K_{2,0}(1) & K_{2,1}(1) & K_{2,1}(0) & K_{2,2}(0)
\end{array}\right| \\
& =\beta_{2} \operatorname{det}\left|\begin{array}{cccc}
K_{0,0}(0) & K_{0,1}(0) & K_{0,1}(-1) & K_{0,2}(-1) \\
K_{1,0}(0) & K_{1,1}(0)-1 & K_{1,1}(-1) & K_{1,2}(-1) \\
K_{1,0}(1) & K_{1,1}(1) & K_{1,1}(0) & K_{1,2}(0) \\
K_{2,0}(1) & K_{2,1}(1) & K_{2,1}(0) & K_{2,2}(0)
\end{array}\right| .
\end{aligned}
$$

Due to the relation (21), applied to the first determinant,

$$
\operatorname{det}\left|\begin{array}{cccc}
K_{0,0}(0) & K_{0,1}(0) & \beta_{1} K_{0,1}(-1) & K_{0,2}(-1) \\
K_{1,0}(0) & K_{1,1}(0) & \beta_{1} K_{1,1}(-1) & K_{1,2}(-1) \\
K_{1,0}(1) & K_{1,1}(1) & \beta_{1}\left[K_{1,1}(0)-1\right] & K_{1,2}(0) \\
K_{2,0}(1) & K_{2,1}(1) & \beta_{1} K_{2,1}(0) & K_{2,2}(0)
\end{array}\right|
$$

subtraction from the third column the first one and adding the second one, results in

$\operatorname{det}\left|\begin{array}{cccc}K_{0,0}(0) & K_{0,1}(0) & 0 & K_{0,2}(-1) \\ K_{1,0}(0) & K_{1,1}(0) & 1 & K_{1,2}(-1) \\ K_{1,0}(1) & K_{1,1}(1) & 0 & K_{1,2}(0) \\ K_{2,0}(1) & K_{2,1}(1) & 0 & K_{2,2}(0)\end{array}\right|=-\operatorname{det}\left|\begin{array}{ccc}K_{0,0}(0) & K_{0,1}(0) & K_{0,2}(-1) \\ K_{1,0}(1) & K_{1,1}(1) & K_{1,2}(0) \\ K_{2,0}(1) & K_{2,1}(1) & K_{2,2}(0)\end{array}\right|$.

Due to the relation (20), applied to the second determinant,

$$
\operatorname{det}\left|\begin{array}{cccc}
K_{0,0}(0) & K_{0,1}(0) & K_{0,1}(-1) & K_{0,2}(-1) \\
\beta_{2} K_{1,0}(0) & \beta_{2}\left[K_{1,1}(0)-1\right] & \beta_{2} K_{1,1}(-1) & \beta_{2} K_{1,2}(-1) \\
K_{1,0}(1) & K_{1,1}(1) & K_{1,1}(0) & K_{1,2}(0) \\
K_{2,0}(1) & K_{2,1}(1) & K_{2,1}(0) & K_{2,2}(0)
\end{array}\right|
$$


subtraction from the second row the last one and adding the third one, results in:

$$
\begin{aligned}
& \operatorname{det}\left|\begin{array}{cccc}
K_{0,0}(0) & K_{0,1}(0) & K_{0,1}(-1) & K_{0,2}(-1) \\
0 & 0 & 1 & 0 \\
K_{1,0}(1) & K_{1,1}(1) & K_{1,1}(0) & K_{1,2}(0) \\
K_{2,0}(1) & K_{2,1}(1) & K_{2,1}(0) & K_{2,2}(0)
\end{array}\right| \\
& =-\operatorname{det}\left|\begin{array}{lll}
K_{0,0}(0) & K_{0,1}(0) & K_{0,2}(-1) \\
K_{1,0}(1) & K_{1,1}(1) & K_{1,2}(0) \\
K_{2,0}(1) & K_{2,1}(1) & K_{2,2}(0)
\end{array}\right| .
\end{aligned}
$$

But this is the same matrix as above.

2. Now we consider the case $\psi_{1}(u)=\left(1-\alpha_{1} u\right)^{-1}, \psi_{2}(u)=\left(1-\alpha_{2} u\right)^{-1}$. We have to prove that

$$
\alpha_{1} \mathbf{P r}_{K}\left\{\left(\begin{array}{ccc}
0 & * & * \\
* & 1 & 0
\end{array}\right)_{0,1,2}\right\}=\alpha_{2} \mathbf{P r}_{K}\left\{\left(\begin{array}{lll}
0 & 1 & * \\
* & * & 0
\end{array}\right)_{0,1,2}\right\}
$$

or

$$
\begin{array}{r|ccc}
\alpha_{1} \operatorname{det}\left|\begin{array}{ccc}
K_{0,0}(0)-1 & K_{0,1}(1) & K_{0,2}(1) \\
K_{1,0}(-1) & K_{1,1}(0) & K_{1,2}(0) \\
K_{2,0}(-1) & K_{2,1}(0) & K_{2,2}(0)-1
\end{array}\right| \\
=\alpha_{2} \operatorname{det}\left|\begin{array}{ccc}
K_{0,0}(0)-1 & K_{0,1}(0) & K_{0,2}(1) \\
K_{1,0}(0) & K_{1,1}(0) & K_{1,2}(1) \\
K_{2,0}(-1) & K_{2,1}(-1) & K_{2,2}(0)-1
\end{array}\right|
\end{array}
$$

Applying the relation (14) to the first two rows of the first determinant, we see that

$$
\begin{array}{r}
\operatorname{det}\left|\begin{array}{ccc}
K_{0,0}(0)-1 & K_{0,1}(1) & K_{0,2}(1) \\
\alpha_{1} K_{1,0}(-1) & \alpha_{1} K_{1,1}(0) & \alpha_{1} K_{1,2}(0) \\
K_{2,0}(-1) & K_{2,1}(0) & K_{2,2}(0)-1
\end{array}\right| \\
=\operatorname{det}\left|\begin{array}{ccc}
K_{0,0}(0)-1 & K_{0,1}(1) & K_{0,2}(1) \\
K_{1,0}(0) & K_{1,1}(1) & K_{1,2}(1) \\
K_{2,0}(-1) & K_{2,1}(0) & K_{2,2}(0)-1
\end{array}\right| .
\end{array}
$$

Applying now the relation (15) to the second and third columns of the second determinant, we see the same result: 


$$
\begin{aligned}
& \operatorname{det}\left|\begin{array}{ccc}
K_{0,0}(0)-1 & \alpha_{2} K_{0,1}(0) & K_{0,2}(1) \\
K_{1,0}(0) & \alpha_{2} K_{1,1}(0) & K_{1,2}(1) \\
K_{2,0}(-1) & \alpha_{2} K_{2,1}(-1) & K_{2,2}(0)-1
\end{array}\right| \\
& =\operatorname{det}\left|\begin{array}{ccc}
K_{0,0}(0)-1 & K_{0,1}(1) & K_{0,2}(1) \\
K_{1,0}(0) & K_{1,1}(1) & K_{1,2}(1) \\
K_{2,0}(-1) & K_{2,1}(0) & K_{2,2}(0)-1
\end{array}\right| .
\end{aligned}
$$

3. We go to the case $\psi_{1}(u)=\left(1+\beta_{1} u\right), \psi_{2}(u)=\left(1-\alpha_{2} u\right)^{-1}$. Here we need to see that

$$
\beta_{1} \operatorname{Pr}_{K}\left\{\left(\begin{array}{ccc}
* & * & * \\
1 & 1 & 0
\end{array}\right)_{0,1,2}\right\}=\alpha_{2} \operatorname{Pr}_{K}\left\{\left(\begin{array}{ccc}
* & 1 & * \\
1 & * & 0
\end{array}\right)_{0,1,2}\right\},
$$

which is the same as

$$
\beta_{1} \operatorname{Pr}_{K}\left\{\left(\begin{array}{ccc}
* & 0 & * \\
1 & * & 0
\end{array}\right)_{0,1,2}\right\}=\alpha_{2} \operatorname{Pr}_{K}\left\{\left(\begin{array}{ccc}
* & 1 & * \\
1 & * & 0
\end{array}\right)_{0,1,2}\right\} .
$$

(The equivalence of the two identities follows from the simplest case of the interlacing property proved in the previous section.)

Expressed via determinants, this is the relation

$$
\begin{array}{r}
\beta_{1} \operatorname{det}\left|\begin{array}{ccc}
K_{0,0}(0) & K_{0,1}(-1) & K_{0,2}(0) \\
K_{1,0}(1) & K_{1,1}(0)-1 & K_{1,2}(1) \\
K_{2,0}(0) & K_{2,1}(-1) & K_{2,2}(0)-1
\end{array}\right| \\
=-\alpha_{2} \operatorname{det}\left|\begin{array}{ccc}
K_{0,0}(0) & K_{0,1}(-1) & K_{0,2}(0) \\
K_{1,0}(1) & K_{1,1}(0) & K_{1,2}(1) \\
K_{2,0}(0) & K_{2,1}(-1) & K_{2,2}(0)-1
\end{array}\right| .
\end{array}
$$

By (21), subtracting in the first determinant,

$$
\operatorname{det}\left|\begin{array}{ccc}
K_{0,0}(0) & \beta_{1} K_{0,1}(-1) & K_{0,2}(0) \\
K_{1,0}(1) & \beta_{1}\left[K_{1,1}(0)-1\right] & K_{1,2}(1) \\
K_{2,0}(0) & \beta_{1} K_{2,1}(-1) & K_{2,2}(0)-1
\end{array}\right|
$$

the first column from the second one, makes it into

$$
\operatorname{det}\left|\begin{array}{ccc}
K_{0,0}(0) & -K_{0,1}(0) & K_{0,2}(0) \\
K_{1,0}(1) & -K_{1,1}(1) & K_{1,2}(1) \\
K_{2,0}(0) & -K_{2,1}(0) & K_{2,2}(0)-1
\end{array}\right|
$$


From (15), adding in the second determinant,

$$
-\operatorname{det}\left|\begin{array}{ccc}
K_{0,0}(0) & \alpha_{2} K_{0,1}(-1) & K_{0,2}(0) \\
K_{1,0}(1) & \alpha_{2} K_{1,1}(0) & K_{1,2}(1) \\
K_{2,0}(0) & \alpha_{2} K_{2,1}(-1) & K_{2,2}(0)-1
\end{array}\right|
$$

the third column to the second one results in

$$
-\operatorname{det}\left|\begin{array}{ccc}
K_{0,0}(0) & K_{0,1}(0) & K_{0,2}(0) \\
K_{1,0}(1) & K_{1,1}(1) & K_{1,2}(1) \\
K_{2,0}(0) & K_{2,1}(0) & K_{2,2}(0)-1
\end{array}\right|
$$

which is what we need.

4. The remaining case is $\psi_{1}(u)=\left(1-\alpha_{1} u\right)^{-1}, \psi_{2}(u)=\left(1+\beta_{2} u\right)$. Here we need to see that

$$
\alpha_{1} \operatorname{Pr}_{K}\left\{\left(\begin{array}{ccc}
0 & * & 1 \\
* & 1 & *
\end{array}\right)_{0,1,2}\right\}=\beta_{2} \operatorname{Pr}_{K}\left\{\left(\begin{array}{lll}
0 & 1 & 1 \\
* & * & *
\end{array}\right)_{0,1,2}\right\},
$$

which is the same as

$$
\alpha_{1} \operatorname{Pr}_{K}\left\{\left(\begin{array}{ccc}
0 & * & 1 \\
* & 1 & *
\end{array}\right)_{0,1,2}\right\}=\beta_{2} \operatorname{Pr}_{K}\left\{\left(\begin{array}{lll}
0 & * & 1 \\
* & 0 & *
\end{array}\right)_{0,1,2}\right\} .
$$

The determinant relation to be checked is

$$
\begin{aligned}
& -\alpha_{1} \operatorname{det}\left|\begin{array}{ccc}
K_{0,0}(0)-1 & K_{0,1}(1) & K_{0,2}(0) \\
K_{1,0}(-1) & K_{1,1}(0) & K_{1,2}(-1) \\
K_{2,0}(0) & K_{2,1}(1) & K_{2,2}(0)
\end{array}\right| \\
& =\beta \operatorname{det}\left|\begin{array}{ccc}
K_{0,0}(0)-1 & K_{0,1}(1) & K_{0,2}(0) \\
K_{1,0}(-1) & K_{1,1}(0)-1 & K_{1,2}(-1) \\
K_{2,0}(0) & K_{2,1}(1) & K_{2,2}(0)
\end{array}\right| .
\end{aligned}
$$

By (14), applied to the first determinant,

$$
-\operatorname{det}\left|\begin{array}{ccc}
K_{0,0}(0)-1 & K_{0,1}(1) & K_{0,2}(0) \\
\alpha_{1} K_{1,0}(-1) & \alpha_{1} K_{1,1}(0) & \alpha_{1} K_{1,2}(-1) \\
K_{2,0}(0) & K_{2,1}(1) & K_{2,2}(0)
\end{array}\right|
$$

the addition of the first row to the second one makes it into

$$
-\operatorname{det}\left|\begin{array}{ccc}
K_{0,0}(0)-1 & K_{0,1}(1) & K_{0,2}(0) \\
K_{1,0}(0) & K_{1,1}(1) & K_{1,2}(0) \\
K_{2,0}(0) & K_{2,1}(1) & K_{2,2}(0)
\end{array}\right|
$$


Applying (20) to the determinant

$$
\operatorname{det}\left|\begin{array}{ccc}
K_{0,0}(0)-1 & K_{0,1}(1) & K_{0,2}(0) \\
\beta_{2} K_{1,0}(-1) & \beta_{2} K_{1,1}(0)-1 & \beta_{2} K_{1,2}(-1) \\
K_{2,0}(0) & K_{2,1}(1) & K_{2,2}(0)
\end{array}\right|
$$

we turn it, after subtracting the third row from the second one, into

$$
\operatorname{det}\left|\begin{array}{ccc}
K_{0,0}(0)-1 & K_{0,1}(1) & K_{0,2}(0) \\
-K_{1,0}(0) & -K_{1,1}(1) & -K_{1,2}(0) \\
K_{2,0}(0) & K_{2,1}(1) & K_{2,2}(0)
\end{array}\right|
$$

That finishes our proof.

\subsection{Moves in general environment}

Now we have to check that the identities of the previous subsection holds in more general situation. For the $\beta \beta$ case it means for example that

$$
\beta_{1} \operatorname{Pr}_{K}\left\{\left(\begin{array}{ccc}
* & 0 & 1 \\
1 & 1 & *
\end{array}\right)_{0,1,2} \cup n_{V}\right\}=\beta_{2} \operatorname{Pr}_{K}\left\{\left(\begin{array}{ccc}
* & 1 & 1 \\
1 & 0 & *
\end{array}\right)_{0,1,2} \cup n_{V}\right\},
$$

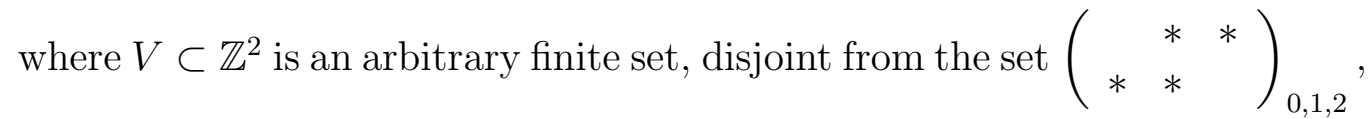
and the symbol $\left\{\left(\begin{array}{ccc}* & 0 & 1 \\ 1 & 1 & *\end{array}\right)_{0,1,2} \cup n_{V}\right\}$ denotes the event that we have the configuration $\left(\begin{array}{ccc}* & 0 & 1 \\ 1 & 1 & *\end{array}\right)_{0,1,2}$ in our window, and all the sites in $V$ are occupied by the particles. Consider the case when $V$ is just a single site $(\zeta, z) \in \mathbb{Z}^{2}$. Let us see that the same relations which were used in the subsection 3.3, work here as well. 
We need to show that

$$
\begin{aligned}
& \beta_{1} \operatorname{det}\left|\begin{array}{ccccc}
K_{0,0}(0) & K_{0,1}(0) & K_{0,1}(-1) & K_{0,2}(-1) & K_{0, \zeta}(-z) \\
K_{1,0}(0) & K_{1,1}(0) & K_{1,1}(-1) & K_{1,2}(-1) & K_{1, \zeta}(-z) \\
K_{1,0}(1) & K_{1,1}(1) & K_{1,1}(0)-1 & K_{1,2}(0) & K_{1, \zeta}(1-z) \\
K_{2,0}(1) & K_{2,1}(1) & K_{2,1}(0) & K_{2,2}(0) & K_{2, \zeta}(1-z) \\
K_{\zeta, 0}(z) & K_{\zeta, 1}(z) & K_{\zeta, 1}(z-1) & K_{\zeta, 2}(z-1) & K_{\zeta, \zeta}(0)
\end{array}\right| \\
& =\beta_{2} \operatorname{det}\left|\begin{array}{ccccc}
K_{0,0}(0) & K_{0,1}(0) & K_{0,1}(-1) & K_{0,2}(-1) & K_{0, \zeta}(-z) \\
K_{1,0}(0) & K_{1,1}(0)-1 & K_{1,1}(-1) & K_{1,2}(-1) & K_{1, \zeta}(-z) \\
K_{1,0}(1) & K_{1,1}(1) & K_{1,1}(0) & K_{1,2}(0) & K_{1, \zeta}(1-z) \\
K_{2,0}(1) & K_{2,1}(1) & K_{2,1}(0) & K_{2,2}(0) & K_{2, \zeta}(1-z) \\
K_{\zeta, 0}(z) & K_{\zeta, 1}(z) & K_{\zeta, 1}(z-1) & K_{\zeta, 2}(z-1) & K_{\zeta, \zeta}(0)
\end{array}\right| .
\end{aligned}
$$

It is immediate to see that the same strategy which was used in the simplest case $4 \times 4$ works: application of (21) turns the determinant

$$
\operatorname{det}\left|\begin{array}{ccccc}
K_{0,0}(0) & K_{0,1}(0) & \beta_{1} K_{0,1}(-1) & K_{0,2}(-1) & K_{0, \zeta}(-z) \\
K_{1,0}(0) & K_{1,1}(0) & \beta_{1} K_{1,1}(-1) & K_{1,2}(-1) & K_{1, \zeta}(-z) \\
K_{1,0}(1) & K_{1,1}(1) & \beta_{1}\left[K_{1,1}(0)-1\right] & K_{1,2}(0) & K_{1, \zeta}(1-z) \\
K_{2,0}(1) & K_{2,1}(1) & \beta_{1} K_{2,1}(0) & K_{2,2}(0) & K_{2, \zeta}(1-z) \\
K_{\zeta, 0}(z) & K_{\zeta, 1}(z) & \beta_{1} K_{\zeta, 1}(z-1) & K_{\zeta, 2}(z-1) & K_{\zeta, \zeta}(0)
\end{array}\right|
$$

into

$$
\operatorname{det}\left|\begin{array}{ccccc}
K_{0,0}(0) & K_{0,1}(0) & 0 & K_{0,2}(-1) & K_{0, \zeta}(-z) \\
K_{1,0}(0) & K_{1,1}(0) & 1 & K_{1,2}(-1) & K_{1, \zeta}(-z) \\
K_{1,0}(1) & K_{1,1}(1) & 0 & K_{1,2}(0) & K_{1, \zeta}(1-z) \\
K_{2,0}(1) & K_{2,1}(1) & 0 & K_{2,2}(0) & K_{2, \zeta}(1-z) \\
K_{\zeta, 0}(z) & K_{\zeta, 1}(z) & 0 & K_{\zeta, 2}(z-1) & K_{\zeta, \zeta}(0)
\end{array}\right|
$$

while the rhs of (28) is treated as the rhs of (24). So one sees in this way that the identities of the subsection 3.3 work for all sets $V$ in all the cases. 


\subsection{Interlacing property. General case.}

1. The case $\psi_{1}(u)=\left(1-\alpha_{1} u\right)^{-1}$. We will show now that

$$
\begin{aligned}
& \operatorname{Pr}_{K}\left\{\left(\begin{array}{cc}
1 & * \\
0 & * \\
\ldots & * \\
0 & * \\
1 & *
\end{array}\right)_{0,1}\right\}=\operatorname{Pr}_{K}\left\{\left(\begin{array}{cc}
1 & * \\
0 & 0 \\
\ldots & \ldots \\
0 & 0 \\
1 & 1
\end{array}\right)_{0,1}\right\}+ \\
& +\operatorname{Pr}_{K}\left\{( \begin{array} { c c } 
{ 1 } & { * } \\
{ 0 } & { 0 } \\
{ \cdots } & { \ldots } \\
{ 0 } & { 1 } \\
{ 1 } & { 0 }
\end{array} ) _ { 0 , 1 } \left\{+\ldots+\operatorname{Pr}_{K}\left\{\left(\begin{array}{cc}
1 & * \\
0 & 1 \\
\cdots & \ldots \\
0 & 0 \\
1 & 0
\end{array}\right)_{0,1}\right\},\right.\right.
\end{aligned}
$$

that is, if we have a configuration with two particles, separated by a vertical string of $n-2$ holes, then with probability one the next column to the right has in the lower $n-1$ cells exactly one particle and $n-2$ holes. (So our sum above has $n-1$ terms.) This is the particle interlacing property. We will prove it simultaneously with the complementary (reflected) statement:

$$
\begin{gathered}
\operatorname{Pr}_{K}\left\{\left(\begin{array}{cc}
* & 1 \\
* & 0 \\
\ldots & \ldots \\
* & 0 \\
* & 1
\end{array}\right)_{0,1}\right\}=\operatorname{Pr}_{K}\left\{\left(\left(\begin{array}{cc}
0 & 1 \\
0 & 0 \\
\ldots & \ldots \\
1 & 0 \\
* & 1
\end{array}\right)_{0,1}\right\}+\right. \\
+\ldots+\operatorname{Pr}_{K}\left\{\left(\begin{array}{cc}
0 & 1 \\
1 & 0 \\
\ldots & \ldots \\
0 & 0 \\
* & 1
\end{array}\right)_{0,1}\right\}+\operatorname{Pr}_{K}\left\{\left(\begin{array}{cc}
1 & 1 \\
0 & 0 \\
\ldots & \ldots \\
0 & 0 \\
* & 1
\end{array}\right)_{0,1}\right\} .
\end{gathered}
$$

The proof goes by induction on the length of the aforementioned string of the holes. The case of the empty string - i.e. $n=2$ - was dealt with in Section 3.2. So suppose that we know already the relations (29) and (30) for all $n<k$. Let us prove them for $n=k$.

First we can exclude the case in (29) when we have at least two particles in the second column. Indeed, that means that we have there a pattern 


$$
\left(\begin{array}{cc}
0 & 1 \\
0 & 0 \\
\cdots & \cdots \\
0 & 0 \\
* & 1
\end{array}\right)_{0,1} \text { with the string of holes in the second column of length less }
$$
than $k-2$, which is ruled out by induction hypothesis for (30). The same argument applies to (30).

It remains to show that $\operatorname{Pr}_{K}\left\{\left(\begin{array}{cc}1 & * \\ 0 & 0 \\ \ldots & \ldots \\ 0 & 0 \\ 1 & 0\end{array}\right)_{0,1}\right\}=0$, where we have $k-1$ holes in the right column. Here we note that the above probability depends on $K$ only through one parameter, $\alpha_{1}$. So without loss of generality we can assume that we are in the " $\alpha \alpha$ " situation, i.e. that $\psi_{0}(u)=\left(1-\alpha_{0} u\right)^{-1}$. Let us write our event as a sum of four events:

$$
\begin{aligned}
\left(\begin{array}{cc}
1 & * \\
0 & 0 \\
\cdots & \cdots \\
0 & 0 \\
1 & 0
\end{array}\right)_{0,1} & =\left(\begin{array}{ccc}
1 & 1 & * \\
* & 0 & 0 \\
* & \ldots & \ldots \\
1 & 0 & 0 \\
* & 1 & 0
\end{array}\right)_{-1,0,1}+\left(\begin{array}{ccc}
1 & 1 & * \\
* & 0 & 0 \\
* & \ldots & \ldots \\
0 & 0 & 0 \\
* & 1 & 0
\end{array}\right)_{-1,0,1} \\
& +\left(\begin{array}{ccc}
0 & 1 & * \\
* & 0 & 0 \\
* & \cdots & \cdots \\
1 & 0 & 0 \\
* & 1 & 0
\end{array}\right)_{-1,0,1}+\left(\begin{array}{ccc}
0 & 1 & * \\
* & 0 & 0 \\
* & \cdots & \cdots \\
0 & 0 & 0 \\
* & 1 & 0
\end{array}\right)_{-1,0,1}
\end{aligned}
$$

The first one has zero probability; this is our induction assumption. And to every one of the remaining events we can apply the move transformation (25), which makes the two particles in the middle column to become one unit closer. After that we get a configuration, which has zero probability by induction hypothesis. This ends the proof of our statement. 
2. The case $\psi_{1}(u)=\left(1+\beta_{1} u\right)^{-1}$. We will show that

$$
\begin{aligned}
& \operatorname{Pr}_{K}\{\left.\left(\begin{array}{cc}
0 & * \\
1 & * \\
\ldots & * \\
1 & * \\
0 & *
\end{array}\right)_{0,1}\right\}=\operatorname{Pr}_{K}\left\{\left(\left(\begin{array}{cc}
0 & 0 \\
1 & 1 \\
\ldots & \ldots \\
1 & 1 \\
0 & *
\end{array}\right)_{0,1}\right\}+\right. \\
&+\operatorname{Pr}_{K}\left\{( \begin{array} { c c } 
{ 0 } & { 1 } \\
{ 1 } & { 0 } \\
{ \ldots } & { \ldots } \\
{ 1 } & { 1 } \\
{ 0 } & { * }
\end{array} ) _ { 0 , 1 } \left\{+\ldots+\operatorname{Pr}_{K}\left\{\left(\begin{array}{cc}
0 & 1 \\
1 & 1 \\
\ldots & \ldots \\
1 & 0 \\
0 & *
\end{array}\right)_{0,1}\right\} .\right.\right.
\end{aligned}
$$

Here in the lhs we have the probability of the event that two holes are separated by the string of $n-2$ particles, while in the rhs we have a sum of probabilities of the $n-1$ events that the right column has exactly one hole in the upper $n-1$ positions. This is the hole interlacing. Again, we will prove it by induction on $n$, the case $n=2$ was established above, see (23) . We will treat simultaneously the reflected event as well (compare with (29) and (30).)

The presence of more than one hole in the right column in (32) is again ruled out by induction. To study the probability of the event $\left(\begin{array}{cc}0 & 1 \\ 1 & 1 \\ \ldots & \ldots \\ 1 & 1 \\ 0 & *\end{array}\right)_{0,1}$ we can without loss of generality consider the case $\psi_{0}(u)=\left(1+\beta_{0} u\right)^{-1}$, and 
we write

$$
\begin{aligned}
\left(\begin{array}{cc}
0 & 1 \\
1 & 1 \\
\cdots & \cdots \\
1 & 1 \\
0 & *
\end{array}\right)_{0,1}=\left(\begin{array}{ccc}
* & 0 & 1 \\
0 & 1 & 1 \\
* & \ldots & \ldots \\
* & 1 & 1 \\
0 & 0 & *
\end{array}\right)_{-1,0,1}+\left(\begin{array}{ccc}
* & 0 & 1 \\
1 & 1 & 1 \\
* & \ldots & \ldots \\
* & 1 & 1 \\
0 & 0 & *
\end{array}\right)_{-1,0,1} \\
+\left(\begin{array}{ccc}
* & 0 & 1 \\
0 & 1 & 1 \\
* & \cdots & \ldots \\
* & 1 & 1 \\
1 & 0 & *
\end{array}\right)_{-1,0,1}+\left(\begin{array}{ccc}
* & 0 & 1 \\
1 & 1 & 1 \\
* & \cdots & \cdots \\
* & 1 & 1 \\
1 & 0 & *
\end{array}\right)_{-1,0,1}
\end{aligned}
$$

The first event is ruled out by induction, while the remaining three are movable, and the application of the corresponding move (the first one described in Section 3.3) reduces the length of the string of particles in the middle column (0-th one) by one, so the remaining three events also have vanishing probability.

\subsection{The downward paths.}

We will show now that the case of the functions $\psi$-s of the types $\left(1-\alpha_{k}^{-} u^{-1}\right)^{-1}$ and $\left(1+\beta_{k}^{-} u^{-1}\right)$ can be reduced to the one when all $\psi_{k}(u)$ are of the form $\left(1-\alpha_{k}^{+} u\right)^{-1}$ or $\left(1+\beta_{k}^{+} u\right)$. Indeed, we have the identities

$$
\begin{aligned}
\left(1-\alpha_{k}^{-} u^{-1}\right)^{-1} & =-\alpha_{k}^{-} u\left(1-\left(\alpha_{k}^{-}\right)^{-1} u\right)^{-1}, \\
\left(1+\beta_{k}^{-} u^{-1}\right) & =\beta_{k}^{-} u^{-1}\left(1+\left(\beta_{k}^{-}\right)^{-1} u\right) .
\end{aligned}
$$

Observe that multiplication of $\psi_{k}(u)$ by a constant $c$ leads to the conjugation of the kernel:

$$
K(\sigma, x ; \tau, y) \mapsto\left\{\begin{array}{cc}
c K(\sigma, x ; \tau, y) & \text { if } \sigma \geq k>\tau \\
c^{-1} K(\sigma, x ; \tau, y) & \text { if } \tau \geq k>\sigma \\
K(\sigma, x ; \tau, y) & \text { otherwise }
\end{array}\right.
$$

which does not affect the determinants for the correlation functions. On the other hand, the multiplication of $\psi_{k}(u)$ by $u$ in the formula for the kernel is equivalent to the following transformation of the state space $\mathbb{Z}^{2}$ :

$$
(\sigma, x) \mapsto\left\{\begin{array}{cc}
(\sigma, x) & \text { if } \sigma<k \\
(\sigma, x+1) & \text { if } \sigma \geq k
\end{array}\right.
$$


Under this transformation every configuration which was satisfying the downward interlacing (for particles or for holes) in the column $\{(\sigma, x): \sigma=k, k+1\}$ will satisfy the upward interlacing, so the new configuration can still be associated with a collection of paths. It is straightforward to see that the change of the weight of a path affected by elementary move is the same in both path configurations, compare the definitions (5) , (7), (9) , (11). That proves our statement.

\subsection{The Gibbs property}

Now we are in the position to check the Gibbs property of the field $n$ viewed as the probability distribution over the lattice paths built from the patterns (44), (6) , (8) and (10) . After the preliminary work we did it is almost immediate.

Indeed, we have already checked in the subsections 3.3, 3.4 that the ratio of the probabilities of two configurations $n_{V}^{\prime}$ and $n_{V}^{\prime \prime}$ which differ by allowed move of one particle depends only on the type of the move and equals to the exponent of the action difference for the corresponding paths. Let us take, in particular, any (finite simply-connected) box $\Lambda \subset \mathbb{Z}^{2}$, and fix the sets $P$ and $Q$ of the entrance and exit points of the paths on the boundary $\partial \Lambda$. Note that in that case any allowed configuration of paths in $\Lambda$ can be obtained from any other by a sequence of elementary moves. This claim is essentially obvious; if follows from the fact that there is a minimal path joining any two points (if the set of paths joining the two points is nonempty), and induction on the number of paths. That finishes our proof.

\section{Positivity}

Denote by $\Omega$ the set of elements $\varpi=(\alpha, \beta, \gamma) \in \mathbb{R}_{+}^{\infty} \times \mathbb{R}_{+}^{\infty} \times \mathbb{R}_{+}$such that

$$
\sum_{i=1}^{\infty} \alpha_{i}<\infty, \quad \sum_{i=1}^{\infty} \beta_{i}<\infty .
$$

For $\varpi \in \Omega$, we denote by $\psi_{\varpi}$ the following meromorphic functions on $\mathbb{C}$ :

$$
\psi_{\varpi}(u)=e^{\gamma u} \prod_{j=1}^{\infty} \frac{1+\beta_{j} u}{1-\alpha_{j} u} .
$$


For $\varpi^{+}, \varpi^{-} \in \Omega$, we also set

$$
\psi_{\varpi^{+}, \varpi^{-}}(u)=\psi_{\varpi^{+}}(u) \psi_{\varpi^{-}}\left(u^{-1}\right) .
$$

Coordinates of $\varpi^{ \pm}$will be denotes as $\alpha_{i}^{ \pm}, \beta_{i}^{ \pm}, \gamma^{ \pm}$.

Our goal is to prove the following statement, which is a slight generalization of Theorem 4.4 in [2].

Theorem 2 Fix a complex number $z$ with $\Im z>0$ and denote $C_{ \pm}$any contour that joins $\bar{z}$ and $z$ and crosses the real axis at a point of $\mathbb{R}_{ \pm}$. Then for any doubly infinite sequences $\left\{\varpi^{+}[k], \varpi^{-}[k]\right\}_{k \in \mathbb{Z}}$ of elements in $\Omega$, there exists a (unique) determinantal point process on $\mathbb{Z} \times \mathbb{Z}$ with the correlation kernel

$$
K(\sigma, x ; \tau, y)= \begin{cases}\frac{1}{2 \pi i} \int_{C_{+}}\left(\prod_{k=\sigma+1}^{\tau} \psi_{\varpi^{+}[k], \varpi^{-}[k]}(u)\right)^{-1} \frac{d u}{u^{x-y+1}}, & \sigma \leq \tau, \\ \frac{1}{2 \pi i} \int_{C_{-}} \prod_{k=\tau+1}^{\sigma} \psi_{\varpi^{+}[k], \varpi^{-}[k]}(u) \frac{d u}{u^{x-y+1}}, & \sigma>\tau .\end{cases}
$$

Comments. 1. The kernels considered in the previous sections are the ones with each of $\psi_{\varpi^{+}[k], \varpi^{-}[k]}(u)$ having the form either $\left(1-\alpha_{k} u\right)^{-1}$ or $\left(1+\beta_{k} u\right)$.

2. The classical fact that lies at the foundation of this theorem is that functions $\psi_{\varpi}(u)$ are generating functions of the totally positive sequences. This statement was independently proved by Aissen-Edrei-Schoenberg-Whitney in 1951 [1], [7], and by Thoma in 1964 [17]. An excellent exposition of deep relations of this result to representation theory of the infinite symmetric group can be found in Kerov's book [11].

3. The equal time restriction of the kernel above is equivalent to the discrete sine kernel on $\mathbb{Z}$; for any $\tau \in \mathbb{Z}$

$$
K(\tau, x ; \tau, y)=\frac{1}{2 \pi i} \int_{C_{+}} \frac{d \zeta}{\zeta^{x-y+1}}=\frac{e^{|z| y}}{e^{|z| x}} \frac{\sin ((\arg z)(x-y))}{\pi(x-y)} .
$$

In particular, the density of particles is equal to $\arg z / \pi$ everywhere. The kernels $K(\sigma, x ; \tau, y)$ may be viewed as extensions of the discrete sine kernel.

4. The class of the random point processes afforded by this theorem is closed under 
- projections of $\mathbb{Z} \times \mathbb{Z}$ to $A \times \mathbb{Z}$, where $A=\left\{a_{n}\right\}_{n=-\infty}^{+\infty}$ is any doubly infinite sequence of integers;

- shifts and reflection of either of the two coordinates of $\mathbb{Z} \times \mathbb{Z}$;

- particle-hole inversion on any subset of the form $B \times \mathbb{Z}$, where $B \subset \mathbb{Z}$.

5 . The projection of the process to the set $\{1, \ldots, T\} \times \mathbb{Z}$ depend only on $\varpi^{ \pm}[k]$ with $k=1, \ldots, T$.

We will give two proofs of the theorem; one is essentially a reduction to Theorem 4.4 from [2], while the second one is "more constructive" - it explains how to build our process from a deformation of the uniform measure on large plane partitions.

Proof 1. Observe that the change of the integration variable $u=r v, r>$ 0 , replaces the formula for the kernel by a similar one with $z \mapsto z / r$, all coordinates of $\varpi_{k}^{+}$'s multiplied by $r$, all coordinates of $\varpi_{k}^{-}$'s divided by $r$, and the integral itself multiplied by $r^{y-x}$. The prefactor $r^{y-x}$ cancels out in the determinants of the form $\operatorname{det}\left[K\left(\sigma_{i}, x_{i} ; \sigma_{j}, x_{j}\right)\right]$, thus it can be removed. Hence, it suffices to prove the claim for $z$ with $|z|=1$.

Observe further, that multiplication of $\psi_{\varpi^{+}[m], \varpi^{-}[m]}(u)$ by $u^{n}$ in the formula for the kernel above is equivalent to the following transformation of the state space $\mathbb{Z} \times \mathbb{Z}$ :

$$
(\sigma, x) \mapsto \begin{cases}(\sigma, x), & \sigma<m, \\ (\sigma, x+n), & \sigma \geq m .\end{cases}
$$

On the other hand, multiplication of $\psi_{\varpi^{+}[m], \varpi^{-}[m]}(u)$ by a constant $c$ leads to the conjugation of the kernel

$$
K(\sigma, x ; \tau, y) \mapsto \begin{cases}c K(\sigma, x ; \tau, y), & \sigma+1 \leq m \leq \tau, \\ c^{-1} K(\sigma, x ; \tau, y), & \tau+1 \leq m \leq \sigma, \\ K(\sigma, x ; \tau, y), & \text { otherwise, }\end{cases}
$$

which does not affect the determinants for the correlation functions.

The identities

$$
1-\alpha u=-\alpha u \cdot\left(1-\alpha^{-1} u^{-1}\right), \quad 1+\beta u=\beta u \cdot\left(1+\beta^{-1} u^{-1}\right)
$$

then show that we can freely replace parameters $\alpha_{i}^{+}[k]=\alpha$ and $\beta_{i}^{+}[k]=\beta$ by $\alpha_{i}^{-}[k]=\alpha^{-1}$ and $\beta_{i}^{-}[k]=\beta^{-1}$ and the other way around, and such changes do not affect the statement that the kernel defines a random point process. 
Using such replacements we can then choose the parameters in such a way that all $\alpha_{i}^{ \pm}[k], \beta_{i}^{ \pm}[k]$ are in the segment $[0,1]$. Since the statement of the theorem is stable under limit transitions, we can assume that all the parameters are strictly smaller than 1 without loss of generality.

But if the parameters satisfy the conditions

$$
|z|=1, \quad \alpha_{i}^{ \pm}[k], \beta_{i}^{ \pm}[k]<1, \quad \text { for all } i, k
$$

then our claim is exactly Theorem 4.4 in [2].

Proof 2. The argument is based on the Schur process of [13] and can be constructed as follows. We use the definitions and notation of [13].

Let us construct a deformation of the Schur process. More precisely, the Schur process is parameterized by two sequences $\left\{\phi^{+}[m], \phi^{-}[m]\right\}_{m \in \mathbb{Z}+\frac{1}{2}}$ of functions holomorphic and nonvanishing in some neighborhood of the interior (resp., exterior) of the unit disc. In order for the process to assign positive weights, the functions $\phi^{ \pm}$have to be such that all minors of the triangular Toeplitz matrices with symbols $\phi^{+}(u)$ and $\phi^{-}\left(u^{-1}\right)$ are nonnegative; this is exactly the content of Comment 2 above.

The concrete example of the Schur process studied asymptotically in [13 corresponds to the choice

$$
\begin{array}{r}
\phi^{+}[m](u)= \begin{cases}\left(1-q^{-m} u\right)^{-1}, & m<0, \\
1, & m>0\end{cases} \\
\phi^{-}[m](u)= \begin{cases}1, & m<0 \\
\left(1-q^{m} u^{-1}\right)^{-1}, & m>0 .\end{cases}
\end{array}
$$

Let us choose $N$ consecutive values of $m$, say $M, M+1, \ldots, M+N-1$, and replace the corresponding functions $\phi^{ \pm}$as follows:

$$
\widetilde{\phi}^{+}[M+k](u)=\psi_{\varpi^{+}[k]}(u), \quad \widetilde{\phi}^{-}[M+k](u)=\psi_{\varpi^{-}[k]}\left(u^{-1}\right),
$$

for $k=0, \ldots, N-1$.

Taking the point of the limit shape with $\tau=0$ (near the corner), one readily sees that such a modification produces no impact on the asymptotic analysis of [13] until the very last stage - the computation of the residue denoted as $\int^{(2)}$ in Section 3.1.6. 
The residue is an integral of the expression in formula (26) without the factor $(z-w)$ in the denominator and with $z=w$, where $\Phi(t, z)$ is defined by the formula (20). The computation gives

$$
\frac{1}{2 \pi i} \int_{\bar{z}_{c}}^{z_{c}} \prod_{m=t_{j}+1}^{t_{i}} \frac{1}{\phi^{+}[m]\left(w^{-1}\right) \phi^{-}[m]\left(w^{-1}\right)} \frac{d w}{w^{h_{i}-h_{j}+\left(t_{i}-t_{j}\right) / 2+1}}
$$

for $t_{i} \geq t_{j}$ and

$$
-\frac{1}{2 \pi i} \int_{z_{c}}^{\bar{z}_{c}} \prod_{m=t_{i}+1}^{t_{j}} \phi^{+}[m]\left(w^{-1}\right) \phi^{-}[m]\left(w^{-1}\right) \frac{d w}{w^{h_{i}-h_{j}+\left(t_{i}-t_{j}\right) / 2+1}}
$$

for $t_{i}<t_{j}$. If we now choose $M$ in such a way that $t_{i}$ and $t_{j}$ lie in the set $M, M+1, \ldots, M+N-1$ then substituting the deformed functions $\widetilde{\phi}^{ \pm}[M+$ $k](u)$ we arrive at the kernel (34) with the change of variables

$$
w \mapsto u^{-1}, \quad z_{c} \mapsto z^{-1}, \quad\left(t_{i}, t_{j}\right) \mapsto(\tau, \sigma), \quad\left(h_{i}+\frac{t_{i}}{2}, h_{j}+\frac{t_{j}}{2}\right) \mapsto(y, x) .
$$

Thus, we showed that determinants made from the kernel (34) are limits of the correlation functions of certain point processes.

\section{References}

[1] Aissen, M., Edrei, A., Schoenberg, I. J. , Whitney, A. (1951) On the Generating Functions of Totally Positive Sequences Proc. Natl. Acad. Sci. USA 37, no. 5, 303-307.

[2] Borodin, A. (2006) Periodic Schur process and cylindric partitions. Duke Math. J. 140, no. 3, 391-468.

[3] Borodin, A. , Okounkov, A. and Olshanski, G. (2000) Asymptotics of Plancherel measures for symmetric groups J. Amer. Math. Soc. 13, 491-515.

[4] Burton, R. and Pemantle, R. (1993) Local characteristics, entropy and limit theorems for spanning trees and domino tilings via transferimpedances. Ann. Probab. 21, no. 3, 1329-1371. 
[5] Cerf, R. and Kenyon, R. (2001) The low-temperature expansion of the Wulff crystal in the 3D Ising model. Comm. Math. Phys. 222, $147-179$.

[6] Cohn, H., Kenyon, R., Propp, J. (2001) A variational principle for domino tilings. J. Amer. Math. Soc. 14, no. 2, 297-346.

[7] Edrei, A. (1953) On the generating function of a doubly-infinite, totally positive sequence. Trans. Amer. Math. Soc. 74, no. 3, 367-383.

[8] Johansson, K. (2002) Non-intersecting paths, random tilings and random matrices. Probab. Theory Related Fields 123, no. 2, 225-280.

[9] Johansson, K. (2005) The arctic circle boundary and the Airy process Ann. Probab. 33, 1-30.

[10] Kenyon, R. (1997) Local statistics of lattice dimers. Ann. Inst. H. Poincaré Probab. Statist. 33, no. 5, 591-618.

[11] Kerov, S. V. (2003) Asymptotic Representation Theory of the Symmetric Group and its Applications in Analysis Translations of mathematical monographs 219, Amer. Math. Soc., Providence, RI.

[12] Luby, M., Randall, D. and Sinclair, A.J. (2001) Markov chain algorithms for planar lattice structures SIAM Journal on Computing 31, 167-192.

[13] Okounkov, A. and Reshetikhin, N. (2003) Correlation function of Schur process with application to local geometry of a random 3dimensional Young diagram. J. Amer. Math. Soc. 16, no. 3, 581-603.

[14] Sheffield, S. (2005) Random Surfaces. Astérisque 304.

[15] Shlosman, S. (2000) Geometric variational problems of statistical mechanics and of combinatorics. Probabilistic techniques in equilibrium and nonequilibrium statistical physics. J. Math. Phys. 41, no.3, 13641370 .

[16] Shlosman, S. (2001) The Wulff construction in statistical mechanics and in combinatorics. Russ. Math. Surv., 56, no. 4, 709-738. 
[17] Thoma, E. (1964) Die unzerlegbaren, positive-definiten Klassenfunktionen der abzählbar unendlichen, symmetrischen Gruppe Math. Zeitschr. 85, 40-61. 